\title{
What drives superconductivity in twisted bilayer graphene?
}

1. The interplay of insulating and superconducting orders in magicangle graphene bilayers

Authors: Petr Stepanov, Ipsita Das, Xiaobo Lu, Ali Fahimniya, Kenji

Watanabe, Takashi Taniguchi, Frank H. L. Koppens, Johannes Lischner, Leonid

Levitov, Dmitri K. Efetov

https://arxiv.org/abs/1911.09198

2. Decoupling superconductivity and correlated insulators in twisted bilayer graphene

Authors: Yu Saito, Jingyuan Ge, Kenji Watanabe, Takashi Taniguchi, and Andrea F. Young

https://arxiv.org/abs/1911.13302

3. Tuning electron correlation in magic-angle twisted bilayer graphene using Coulomb screening

Authors: Xiaoxue Liu, Zhi Wang, K. Watanabe, T. Taniguchi, Oskar Vafek, J. I.

A. $\mathrm{Li}$

https://arxiv.org/abs/2003.11072

4. Nematicity and Competing Orders in Superconducting Magic-Angle

Graphene

Authors: Yuan Cao, Daniel Rodan-Legrain, Jeong Min Park, Fanqi Noah

Yuan, Kenji Watanabe, Takashi Taniguchi, Rafael M. Fernandes, Liang Fu, Pablo

Jarillo-Herrero

https://arxiv.org/abs/2004.04148

Recommended with a Commentary by T. Senthil, Massachusetts Institute of Technology, USA.

When two layers of graphene are stacked on top of each other with a small relative twist, a long period moire pattern forms. For the electronic states, this moire superlattice defines a new mini-Brillouin zone determined by the long real space period. Early theory[1] showed that near certain "magic angles" these reconstructed electronic bands have very narrow bandwidths, comparable or lower than the estimated strength of Coulomb interactions between the electrons. This makes twisted bilayer graphene (TBLG) a possible platform for correlated electron physics in a tunable two dimensional material. This expectation was strikingly realized experimentally in 2018 when Pablo-Jarillo Herrero and colleagues discovered insulating states at a partial integer filling of the moire bands[2], and superconductivity[3] when this insulator was doped. This led to a number of dramatic further developments, both in twisted bilayer graphene, and in other moire materials (see, eg, the Journal Club commentary by Zalatel, Feb 2019). 
The initial experiments observing superconductivity[2,4] close to the correlated insulator suggested an analogy to cuprate physics, and led to hopes that similar physics may be operational in these two systems, which microscopically are strikingly different. Recent experimental developments, reported in the highlighted papers, suggest that the story of superconductivity in TBLG may be more complex and fascinating in it's own right.

First in a previous paper[5] Efetov's group made devices (which likely have lower inhomogeneity than previous ones) where the superconductivity appeared in a wide range of doping, punctuated by insulating phases at total integer filling. The highlighted paper by Stepanov et al studies the effect on the TBLG phase diagram of screening the Coulomb interactions (by use of a thin substrate) by a proximate metallic gate. Remarkably the correlated insulators disappeared at partial band filling. However superconductivity survived in a range of densities with Tc as high as $900 \mathrm{mK}$, roughly comparable to what is obtained in devices with thicker substrates where the screening effect is negligible. However the correlated insulator is restored when a small out-of-plane magnetic field of about $0.5 \mathrm{~T}$ is applied. Thus correlation effects are weakened but not completely suppressed.

The paper by Saito et al also presented two TBLG devices where the superconductivity apparently appears in the absence of a correlated insulator. Apart from screening of the Coulomb interaction (one of the devices has a thin $\mathrm{hBN}$ substrate), these devices have twist angles further away from the optimal one for the most narrow bands.

What do we learn from these reports? At face value, it appears that the superconductivity can be decoupled from the correlated insulator. However this conclusion comes with its own mysteries. In the original TBLG phase diagram a prominent superconducting dome occurs at a filling $\boldsymbol{v}_{\mathrm{T}}=-2-\boldsymbol{\delta}$ with $\boldsymbol{\delta}$ small and positive (the filling is defined with respect to charge neutrality; including the spin/valley degree of freedom $\mathbf{v}_{\mathrm{T}}= \pm 4$ correspond to band insulators, and the prominent correlated insulator at fillings $\boldsymbol{v}_{\mathrm{T}}= \pm 2$ ). In all previous experiments[2,3,4,5] quantum oscillation as well as Hall transport experiments show that the normal state carrier density in this region is small and is set by $\boldsymbol{\delta}$. Thus the strong superconductivity at this density range develops out of a normal state with a "small" Fermi surface. As emphasized in the paper by Saito et al, in the devices where the correlated insulator is absent, neither the quantum oscillations nor Hall transport support the picture of a metal with a small Fermi surface. Thus in these devices the normal state out of which the superconductivity develops has - at these fillings - itself been altered in comparison with that in the previous experiments of $[2,3,4,5]$. Clearly then the possibility that, despite some similarities, the superconductivity in these new devices is different from the one in the older ones must be contemplated. 
On the face of it these results points to superconductivity that is more robust than the correlated insulator and complicates attempts to interpret the superconductor as the result of doping a correlated insulator. However the role of disorder (particularly of local variations of twist angle) and other complications must be considered and may be important for understanding some of these results.

The highlighted paper by Liu et al offers a counterpoint to the first two highlighted papers. Liu et al studied a device in which twisted bilayer graphene is separated by a thin $(3 \mathrm{~nm}) \mathrm{hBN}$ layer from ordinary AB-stacked Bilayer Graphene (BLG). The carrier density and density of states of BLG can be tuned by gate voltages, and this in turn enables tuning the the screening of the Coulomb interaction in TBLG within one device. Liu et al found that the correlated insulator of TBLG survived Coulomb screening, albeit with a slightly reduced gap. In contrast the superconductivity was suppressed when screening was turned on.

A result of a different nature is reported in the paper by Cao et al. They found that the critical parallel magnetic field $\mathrm{B}_{/ /}$needed to kill the superconductivity varies as a function of the azimuthal angle $\phi$, tracing out an ellipse rather than a circle. This suggests that the superconducting state breaks 6-fold rotational symmetry expected in an ideal sample. The axes of the ellipse are fixed for a given doping but change as a function of doping. The authors argue that the lack of 6-fold symmetry is an intrinsic property - an example of electronic nematic order similar to what is seen in many interesting correlated superconductors - with anisotropy axes pinned by weak strain or other in-built asymmetry. A different feature of the results of Cao et al is on the anisotropy of the normal state which is probed by measuring the transverse resistance. The anisotropy persists into the normal state up to high temperature only in a narrow range of doping at which the superconducting $\mathrm{T}_{\mathrm{c}}$ has a dip. When the superconductivity is suppressed with a magnetic field, this narrow doping range becomes insulating despite the finite doping away from integer $\boldsymbol{v}_{\mathrm{T}}$. Remarkably this finite-doping insulator is stronger than the nearby one at $\mathbf{v}_{\mathrm{T}}=-2$. The authors suggest density wave formation as a possible explanation. This behavior and the suggested explanation will need to be scrutinized in future work.

These results point to a complex and developing story on the relationship between the superconductor and the correlated insulator in TBLG. This will likely be the focus of much more activity in the future.

\section{References}

1. Rafi Bistritzer and Allan H MacDonald. Moir' e bands in twisted double-layer graphene. Proceedings of the National Academy of Sciences, 108(30):12233, 2011.

2. Yuan Cao et al, Correlated insulator behaviour at half-filling in magic-angle graphene superlattices. Nature, 556(7699):80, 2018.

3. Yuan Cao et al, Nature, 556(7699):43, 2018.

4. Matthew Yankowitz et al, Science, 363, 6431, 1059-1064 (2019).

5. X. Lu et al, Nature, 574, 653 (2019). 